\title{
Reden über Demenz
}

\section{Markus Minder}

Chefarzt Geriatrie und Palliative Care, Spital Affoltern, Mitglied FMH

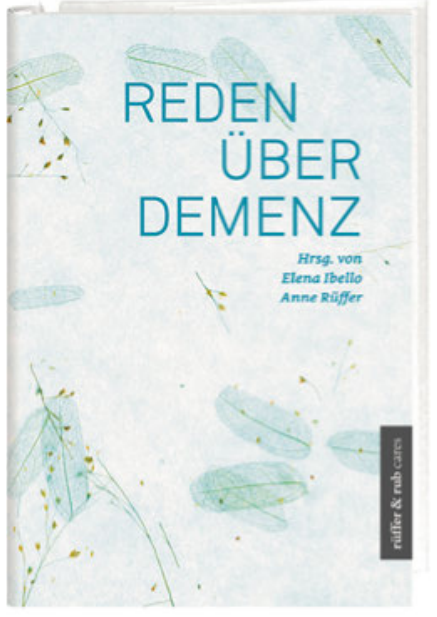

Elena Ibello, Anne Rüffer (Hrsg.)

Reden über Demenz

Zürich: rüffer \& rub; 2017.

168 Seiten. 19.80 CHF.

ISBN 978-3-906304-29-8

Im Oktober letzten Jahres erschien das Buch «Reden über Demenz». Dies ist nach "Reden über Sterben», welches ich ebenfalls wärmstens empfehlen kann, das zweite Buch, das palliative $\mathrm{zh}+\mathrm{sh}$ in Zusammenarbeit mit dem Verlag rüffer \& rub veröffentlicht. Es wurde von der Kommunikationsbeauftragten von palliative $\mathrm{zh}+\mathrm{sh}$ und freien Journalistin Elena Ibello und der Verlegerin und Inhaberin vom Sachbuchverlag rüffer \& rub Anne Rüffer herausgegeben. In «Reden über Demenz» steht die Kunst der Kommunikation mit Demenzkranken und ihren Angehörigen im Fokus. Das Buch vereint Beiträge aus verschiedenen Perspektiven von Fachpersonen wie Frau Dr. med. Irene Bopp-Kistler, Dr. med. Roland Kunz und Prof. Dr. theol. Ralph Kunz und von Betroffenen. Die Diagnose "Demenz" löst Ängste und Krisen aus. Im Buch wird die Wichtigkeit einer korrekten Diagnosestellung aufgezeigt. Wenn eine Demenzdiagnose ausgesprochen wird, folgt Fassungslosigkeit, aber auch eine gewisse Erleichterung. Die Zeit vor der Diagnosestellung ist belastend, da die Symptome, die Fehlleistungen und das merkwürdige Verhalten nicht richtig eingeordnet werden können. Frau Dr. med. Irene Bopp-Kistler schreibt: «Es ist nicht die Diagnose, die die Menschen in den Suizid treibt, sondern es sind die Symptome, die keinen Na- men haben: Es ist die Reaktion des sozialen Umfeldes, das nicht mit den Betroffenen spricht.» Das Buch zeigt auch auf, wie wichtig es ist, eine frühzeitige (ab dem Zeitpunkt der Diagnosestellung) und umfassende Palliative Care den demenzkranken Menschen zukommen zu lassen. Der drohende Verlust der Urteilsfähigkeit macht eine rechtzeitige vorausschauende Planung (Advance Care Planning [ACP]) wichtig, damit frühzeitig über Wertehaltung, Wünsche und Ängste rund um das Lebensende gesprochen werden kann und diese Gedanken und Vorausplanung auch schriftlich festgehalten werden. Diese Vorausplanung ist im Verlauf immer wieder zu evaluieren und gegebenenfalls anzupassen, wie Dr. med. Roland Kunz schreibt. Die rechtzeitig erhobene Werteanamnese soll unter anderem in einem späteren fortgeschrittenen Krankheitsstadium helfen, die individuelle Lebensqualität der Betroffenen zu beurteilen. Das Buch zeigt auch auf, dass Palliative Care nicht nur die Lebensqualität der Betroffenen, sondern auch die Lebensqualität der Angehörigen stützen soll. Der Theologe Prof. Dr. theol. Ralph Kunz beschreibt eindrucksvoll und spannend die theologisch-spirituellen Dimensionen der Erkrankung. «Reden über Demenz» vermittelt zudem Einsichten in die Alltagsgestaltung von demenzbetroffenen Menschen und ihren Angehörigen und bringt uns so das Erleben der Krankheit nahe. Zwei Texte über Menschen, die ihren erkrankten Partner zu Hause betreuen, sowie eine Reportage in einer Demenzwohngruppe zeigen den alltäglichen Umgang mit Demenzerkrankten.

«Reden über Sterben» ist nicht ein Fachbuch im engeren Sinne. Die Sprache ist so gewählt, dass es für ein Laienpublikum verständlich zu lesen ist, wobei das Buch auch für Ärzte und andere Mitarbeiter des Gesundheitswesens äusserst spannend, lehrreich, kurzweilig und empfehlenswert ist. Es vermittelt Fachleuten viele praktische Ideen in der Betreuung von Betroffenen und Angehörigen, übermittelt Hilfreiches in der schwierigen Kommunikation, stellt eine grosse Palette über angemessene Angebote für Betroffene und Angehörige dar, regt uns immer wieder zum Denken an und zeigt uns auf, wo wir in der Betreuung der Betroffenen und der Angehörigen die Prioritäten setzen müssen, um eine hohe Betreuungsqualität zu ermöglichen. Auch als Geschenk eignet sich das Buch hervorragend. 